\title{
From papayas to practice: Surgical simulation and the future of urology training
}

\author{
Keith Francis Rourke, MD, FRCSC \\ Division of Urology, Department of Surgery, University of Alberta, Edmonton, AB
}

See related article on page 32.

Cite as: Can Urol Assoc J 2015;9(1-2):37-8. hitp://dx.doi.org/10.5489/cuaj.2730 Published online February 5, 2015.

I $\mathrm{n}$ light of resident work hour restrictions, decreasing resident caseload, patient expectations, limitations in operative resources and the dawn of competency-based education, there has been a great deal of interest in the field of surgical simulation to supplement resident training. Advocates of surgical simulation strongly feel that now is the time to remove "the learning curve from the operating room." ${ }^{\prime 1}$ This has directly led to initiatives in the United States and the United Kingdom to both develop and mandate national simulation-based curriculum. ${ }^{2,3}$

This manuscript by Nguyen and colleagues ${ }^{4}$ describes a simulation OSCE to assess multiple resident CanMEDS competencies, including medical expert (technical) skills as well as the "intrinsic" roles of CanMEDS communicator, manager, and health advocate. This simulation is a "hybrid" using both low-fidelity and high-fidelity instruments. Residents uniformly agreed that their intrinsic skills were accurately assessed using this approach.

Urology appears well-suited to simulation as a training tool as many of our procedures are either endourologic or laparoscopic. Not surprisingly there are at least 26 different types of endourology simulators available and over 400 published manuscripts describing their face and content validity. ${ }^{5}$ Despite this broad range of simulators, only a handful of manuscripts actually describe the implementation of simulation into a training program. In fact only two manuscripts have further examined the predictive validity of endourology simulation and assessed the correlation between simulation and improvement in operating room performance. Opponents of simulation argue that simulation in urology is only applicable to junior residents learning relatively simple

surgical tasks, such as cystoscopy, and advanced simulation required to recreate complex surgery is not readily available. On balance, it appears that simulation can improve technical skills and shorten the learning curve.

Most would agree that technical skills are fundamentally important for urologists and justifiably much of our focus on resident training has been on the development of technical skills. However, the successful practice of surgery requires more than technical skills alone. Non-technical skills, such as the CanMED's intrinsic roles and surgical decision-making, are likely more important than a surgeon's technical prowess. In fact, a lack of "intrinsic" skills causes more errors in the operating room than deficiencies in technical surgical skill. ${ }^{6}$ Despite the importance of these "intrinsic" skills, it has been difficult to incorporate a robust CanMED's curriculum into residency training. Some feel it is difficult to teach qualities that should be "intrinsic" to being a physician. However, the lack of formal CanMEDs curricula may also be another example of the "tail wagging the dog" in medical education. Learners prioritize what they are examined on. Unless we evaluate and examine CanMEDS roles in a more formal and robust manner, these skills may never be deemed important enough to claim the attention they deserve. Using team-based surgical simulators in our high stakes summative assessments, such as the Royal College exam, may seem controversial, but it is now clear that this type of assessment can be reliably performed. With strong evidence showing the importance of non-medical expert roles in improving surgical outcomes, a pragmatic approach to integrating these skills into our evaluation process is needed. This manuscript represents some of the first steps toward integrating simulation and assessment into a meaningful CanMEDS competency-based urologic curriculum.

Over the next year urology educators from across the country will be faced with the challenge of developing a competency-based curriculum for our residents. Once we establish this core curriculum we will certainly need robust 
Rourke

evaluation tools to assess resident progress and integrating simulation training into this process will likely enhance performance, improve surgical team function and ultimately optimize patient outcomes.

Competing interests: Dr. Rourke declares no competing financial or personal interests.

This paper has been peer-reviewed.

\section{References}

1. Scott DJ, Dunnington GL. The new ACS/APDS skills curriculum: moving the learning curve out of the operating room. J Gastrointest Surg 2008;12:213e21.

2. Shamim Khan $M$, Ahmed K, Gavazzi A, et al. Development and implementation of centralized simulation training: Evaluation of feasibility, acceptability and construct validity. BJU Int 2013;111:518e23.

3. Vassiliou MC, Dunkin BJ, Marks JM, et al. FLS and FES: Comprehensive models of training and assessment. Surg Clin North Am 2010;90:535e58.

4. Nguyen L, Tardioli K, Roberts M, et al. Development and incorporation of hybrid simulation OSCE into in-training examinations to assess multiple CanMEDS competencies in urologic trainees. Can Urol Assoc J 2015;9(1-2):32-6. http://dx.doi.org/10.5489/cuaj.2366

5. Brewin J, Ahmed K, Challacombe B. An update and review of simulation in urological training. Int I Surg 2014;12:103-8. Epub 2013 Dec 4. http://dx.doi.org/10.1016/i.ijs.2013.11.012

6. Rogers Jr SO, Gawande AA, Kwaan $\mathrm{M}$, et al. Analysis of surgical errors in closed malpractice claims at 4 liability insurers. Surgery 2006;140:25e33

Correspondence: Dr. Keith F. Rourke, Associate Professor, Division of Urology, Department of Surgery, University of Alberta, Edmonton, AB; krourke@ualberta.ca 